\title{
Raman scattering of InSb quantum dots grown on InP substrates
}

\author{
G. Armelles, T. Utzmeier, ${ }^{\text {a) }}$ P. A. Postigo, and F. Briones \\ Instituto de Microelectónica de Madrid, CNM-CSIC Isaac Newton 8, P.T.M., Tres Cantos, \\ Madrid E-28760, Spain \\ J. C. Ferrer, P. Peiró, and A. Cornet \\ Enginyeria i Materials Electronics, Dpt. Fisica Aplicada i Electrònica, Universitat de Barcelona, Diagonal \\ 645-647, Barcelona E-08028, Spain
}

(Received 3 September 1996; accepted for publication 6 Janurary 1997)

\begin{abstract}
In this paper we present the Raman scattering of self-assembled InSb dots grown on (001) oriented InP substrates. The samples were grown by pulsed molecular beam epitaxy mode. Two types of samples have been investigated. In one type the InSb dots were capped with 200 monolayers of InP; in the other type no capping was deposited after the InSb dot formation. We observe two peaks in the Raman spectra of the uncapped dot, while only one peak is observed in the Raman spectra of the capped dots. In the case of the uncapped dots the peaks are attributed to LO-like and TO-like vibration of completely relaxed InSb dots, in agreement with high resolution transmission electron microscopy photographs. The Raman spectra of the capped dot suggest a different strain state in the dot due to the capping layer. (C) 1997 American Institute of Physics. [S0021-8979(97)02208-1]
\end{abstract}

\section{INTRODUCTION}

The growth of strained layers on a substrate can lead to the spontaneous formation of clusters. Such spontaneous process can be controlled in order to obtain clusters in the nanometer range, therefore zero-dimensional heterostructures can be fabricated without using technological processes, like lithography and etching. Strong effort has been devoted in the last years to study the growth and properties of these self-assembled quantum dots. These dots have been obtained in systems like $\mathrm{Ga}_{x} \mathrm{In}_{1-x} \mathrm{As} / \mathrm{GaAs}, \mathrm{InSb} / \mathrm{GaAs}$, AlSb/GaAs, GaSb/GaAs, InP/GaInP, and InAs/InP. ${ }^{1-7} \mathrm{De}-$ spite the interest in these nano-structures and the importance that electron-phonon coupling has on device applications, little is known about their phonon properties. ${ }^{8,9}$ In this article we analyse the vibrational properties of InSb dots on InP using Raman scattering. InSb exhibits one of the smallest band-gap of all III-V semiconductors compounds, while InP has a substantially higher band gap and therefore this system is suitable for infrared device applications. Due to the lattice mismatch between the dots and the matrix, strain fields appear. From the Raman measurements information about the strain state inside the dots can be obtained. Such information is important to understand and analyse the electronic properties of the dots. ${ }^{10}$

\section{EXPERIMENT}

The samples were grown by molecular beam epitaxy $(\mathrm{MBE})$ in a pulsed mode, called atomic layer MBE, where only the group V flux is pulsed. The amount of group III and group $\mathrm{V}$ molecules reaching the surface is controlled in situ by means of controlling the surface coverage of In-dimers by the reflection difference method. ${ }^{11}$ A buffer layer of 500 monolayers (MLS) of InP was grown at $410{ }^{\circ} \mathrm{C}$ on (001) oriented semi-insulating InP substrates prior to the $\mathrm{InSb}$

\footnotetext{
a) Author to whom correspondence is to be sent. Electronic mail: thomas@imm.enm.csic.es
}

deposition. The InSb was grown at $410{ }^{\circ} \mathrm{C}$ as well. When more than 1.1 MLS of InSb is deposited on InP we observe the transition from a two-dimensional to a three-dimensional growth mode by means of reflection high energy electron diffraction (RHEED) pattern. The three-dimensional diffraction spots become much better defined if, after the deposition of InSb, an annealing step at $440{ }^{\circ} \mathrm{C}$ for $120 \mathrm{~s}$ under Sb-flux was performed, indicating a much better island formation. The amount of InSb deposition has been varied between 1 and 7 MLS and no material was deposited after the formation of the dots. Only one part of the samples, that is those where the InSb deposition has been varied between 3 and 5 monolayers, were grown with a cap-layer of 200 MLS of InP grown at $410^{\circ} \mathrm{C}$. In the samples with the final cap-layer the RHEED pattern gets streaky again after about 40 MLS.

\section{RESULTS}

According with atomic force microscopy results the dot density in the uncapped samples varies between 1 and $4 \times 10^{10}$ QDs per $\mathrm{cm}^{2}$, depending on the number of $\mathrm{InSb}$ MLS grown and exhibiting a maximum at 2.2 MLS. For this amount of InSb we obtain also the minimum QD-size, with a base width of $24 \pm 4 \mathrm{~nm}$ and a island height of $6 \pm 3 \mathrm{~nm}$. The error values are standard deviations of both distributions. The AFM images show InSb islands with a nearly quadratic base in the range between 1.1 and 3.2 MLS. In that range the maximum dots size obtained corresponds to a deposition of about $3 \mathrm{MLS}$ and the dots have base width of $64 \pm 7 \mathrm{~nm}$ and a height of $11 \pm 2 \mathrm{~nm}$. For InSb deposition of more than 3.2 MLS the islands become rectangular, forming quantum-dashes ${ }^{12}$ with a length-to width ratio up to 2.5 . In this range typical values of the width and height are $40 \mathrm{~nm}$ and $10 \mathrm{~nm}$, respectively.

Several Raman spectra, corresponding to samples with varying amounts of InSb monolayers deposited on the InP substrate, are presented in figure 1. Only the region where features, related to the InSb deposition, appear is presented. The spectra were obtained at $80 \mathrm{~K}$ in the backscattering con- 


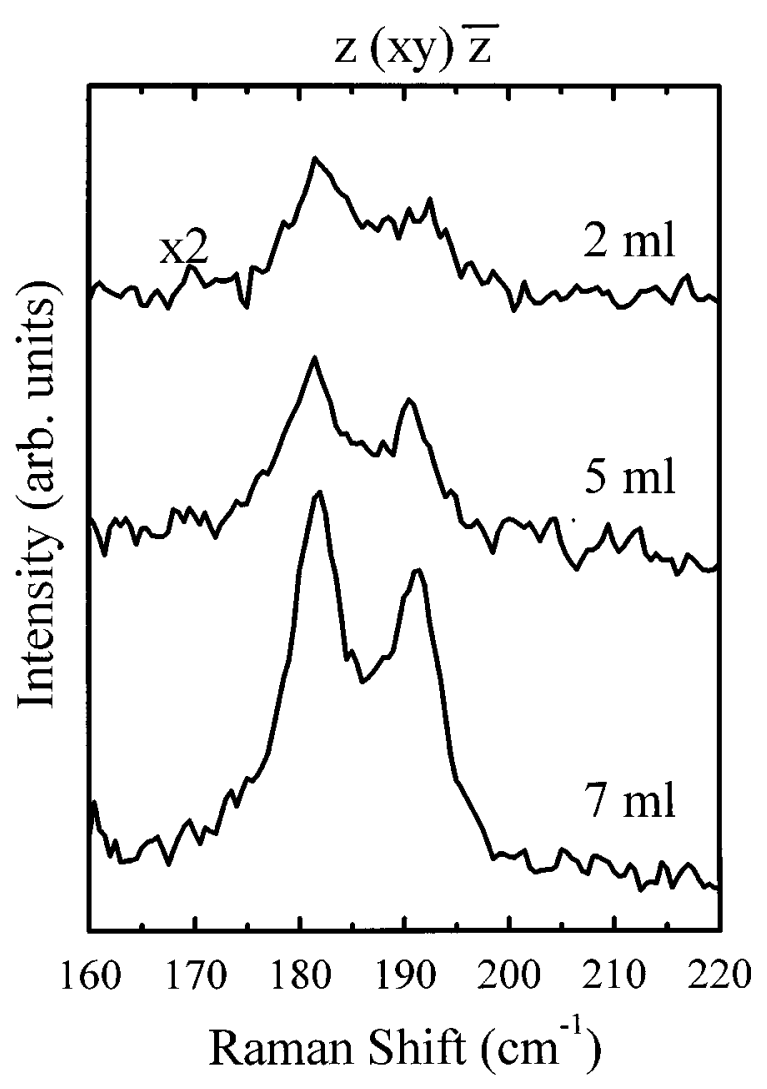

FIG. 1. Raman spectra of samples with 2, 5, and 7 MLs of InSb deposited on the InP surface. Two mode are seen, the one at $193 \mathrm{~cm}^{-1}$ attributed to the LO-like and the other at $181 \mathrm{~cm}^{-1}$ attributed to the TO-like vibration of relaxed $\mathrm{InSb}$.

figuration using the $514.5 \mathrm{~nm}$ line of an $\mathrm{Ar}^{+}$laser. Similar spectra are obtained in the $z(x y) \bar{z}$ and $z(x x) \bar{z}$ configuration being $\{x, y, z\}\{[100],[010],[001]\}$. The spectra are normalised with respect to the InP LO Raman peak. As can be observed two peaks appear in the Raman spectra located at $192 \mathrm{~cm}^{-1}$ and $181 \mathrm{~cm}^{-1}$, respectively. The intensities of these new peaks increase as we increase the amount of InSb deposit on the InP surface, although it does not only depend on the amount of InSb, but also on the growth conditions. In particular, the thermal annealing done to favour the dot formation helps the observation of those peaks. No InSb related signal was detected in samples having an amount of InSb lower than 2 MLS. In particular, no signal was detected in samples where the InSb deposition was lower than 1.1 MLS, which is the onset of dots formation. Therefore, the peaks, observed in the Raman spectra, are attributed to the InSb dots.

\section{DISCUSSION}

The phonon optical branches of InP and InSb do not overlap and confinement effects may appear on the phonons of the InSb dots. Confinement produces a decrease in the frequencies of the modes but, due to the size of the dots, such effect is not expected to be important. ${ }^{13}$ On the other hand the lattice mismatch between $\mathrm{InP}$ and $\mathrm{InSb}$ is very high $(\sim 10 \%)$ and, if the dots are grown coherently on the InP substrates, the strain present in the dots will produce a strong increase of the frequencies of the InSb optical modes. ${ }^{14}$ In agreement with the small confinement effects expected, the frequencies of the Raman peaks are very similar from one sample to the other, despite the different size and shape of the dots, but they are nearly identical to the frequencies of the LO $\left(193 \mathrm{~cm}^{-1}\right)$ and TO $\left(182 \mathrm{~cm}^{-1}\right) \Gamma$ modes of unstrained InSb. ${ }^{15}$ This coincidence strongly suggest that the Raman peaks are due to LO-like $\left(192 \mathrm{~cm}^{-1}\right)$ and TO-like $\left(181 \mathrm{~cm}^{-1}\right)$ vibrations of relaxed InSb dots. This assignment is supported by the photograph of figure 2, where a highresolution transmission electron microscopy (HRTEM) image of a single quantum dot, corresponding to the sample of 5 monolayers, is presented. The HRTEM image reveals a regularly spaced array of pure edge dislocations located at the InSb/InP interface and a good crystalline quality of the $\mathrm{InSb}$ dots. The distances between consecutive dislocations are those required to relieve the strain for the InSb/InP system. Formation of dislocations could also been observed in TEM images, not shown here, of the other samples. Selected area diffraction patterns, figure 3, confirm the high degree of relaxation of the InSb quantum-dots being clearly observable two different diffraction patterns, attributed to the InP bulk and the InSb QDs. The lattice difference in real space corresponds to almost completely relaxed $\mathrm{InSb}$ and $\mathrm{InP}$.

As can be observed in the photograph of figure 2 the dots have the shape of truncated pyramids. The angle of the side walls depends on the direction. Whereas along the [110] direction the side walls we observe $\{111\}$-type planes, for the [110] direction, there are also present $\{113\}$-type planes. The scattering by TO modes is not allowed in the back scattering geometry from the (001) surfaces, but it is allowed for (111) and (113) surfaces. ${ }^{16}$ Due to the difference between the refractive index of InSb and the surrounding uppermost medium a nearly backscattering condition is obtained for the light impinging the side wall planes. Therefore, we suggest that TO modes are observed due to the difference in the refractive index and due to the existence of non-(001) planes in the side walls of the dots. We also suggest that the observation of similar spectra in the $z(x x) \bar{z}$ and $z(x y) \bar{z}$ configurations is also due to the same effect. In the side wall planes the $z(x x) \bar{z}$ (parallel), or $z(x y) \bar{z}$ (orthogonal) geometries of the (001) surfaces are transformed into a combination of parallel and orthogonal geometries of the side wall planes and no clear selection rules can be obtained. Those suggestions are also supported by figure 4, where we present the Raman spectra of two samples, grown under similar conditions and having similar amounts of InSb, but only one has a cap-layer of $200 \mathrm{MLS}$ of InP. According to TEM measurement both samples have dots with similar morphology, but despite of this similarity the Raman spectra are quite different. The sample with the caplayer has a peak at a frequency higher than the peaks of the uncapped sample. Such a peak is only observed in the $z(x y) z$ configuration. We attribute this band to scattering by LO-like phonons of the InSb dots. The shift of this band to higher frequencies with respect of the LO peak of the uncapped dots is due to the strain present in the buried dots. It has been shown that for coherently grown self-assembled quantum dots the strain state inside the dot 


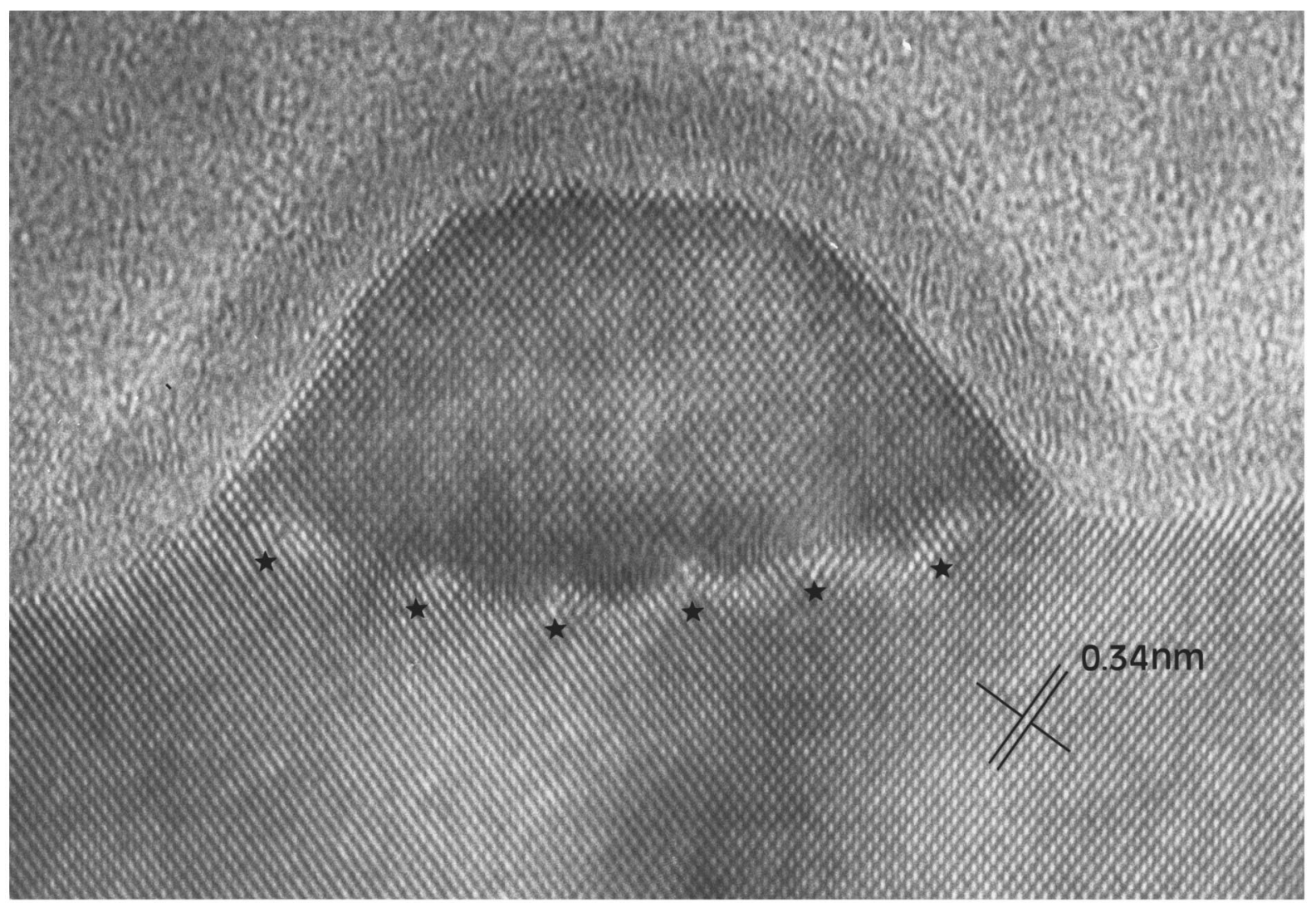

FIG. 2. High-resolution cross-sectional image of an uncapped dot along [110] direction. The stars shown the proximity of a dislocation. Note the lateral facetting with $\{111\}$-type planes and the (001) plane of the top surface.

depends on the cap layer thickness. ${ }^{17}$ In the capped dots the strain in the dots has hydrostatic and biaxial components. The presented dots are not coherently grown and the strain in the buried dots should be more hydrostatic than biaxial, contrary to what has been observed in coherently grown

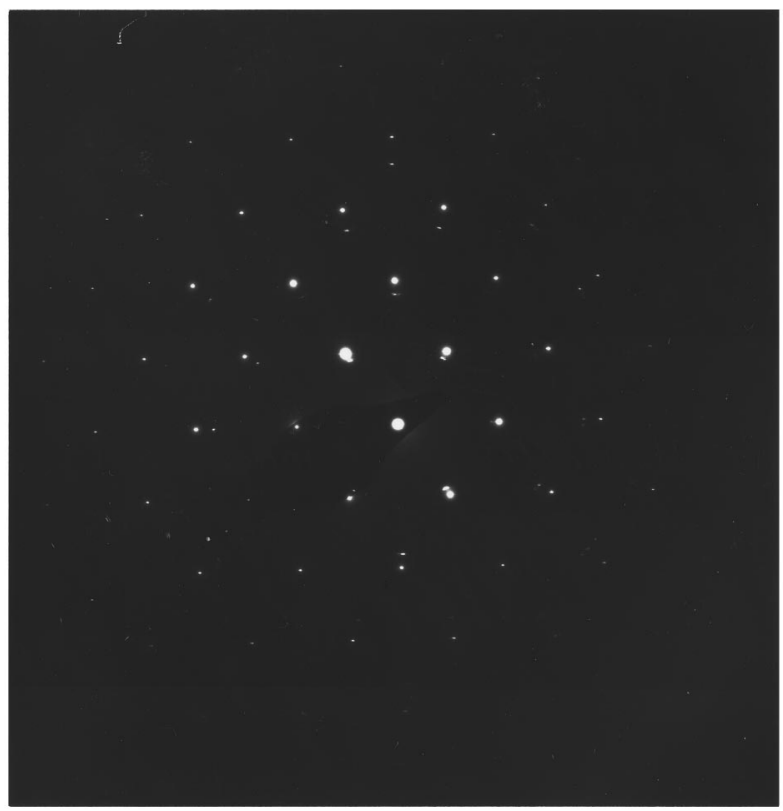

FIG. 3. Diffraction of a region which has various dots and the substrate. Observe the splitting of the spots due to the strain relaxation of the dots.

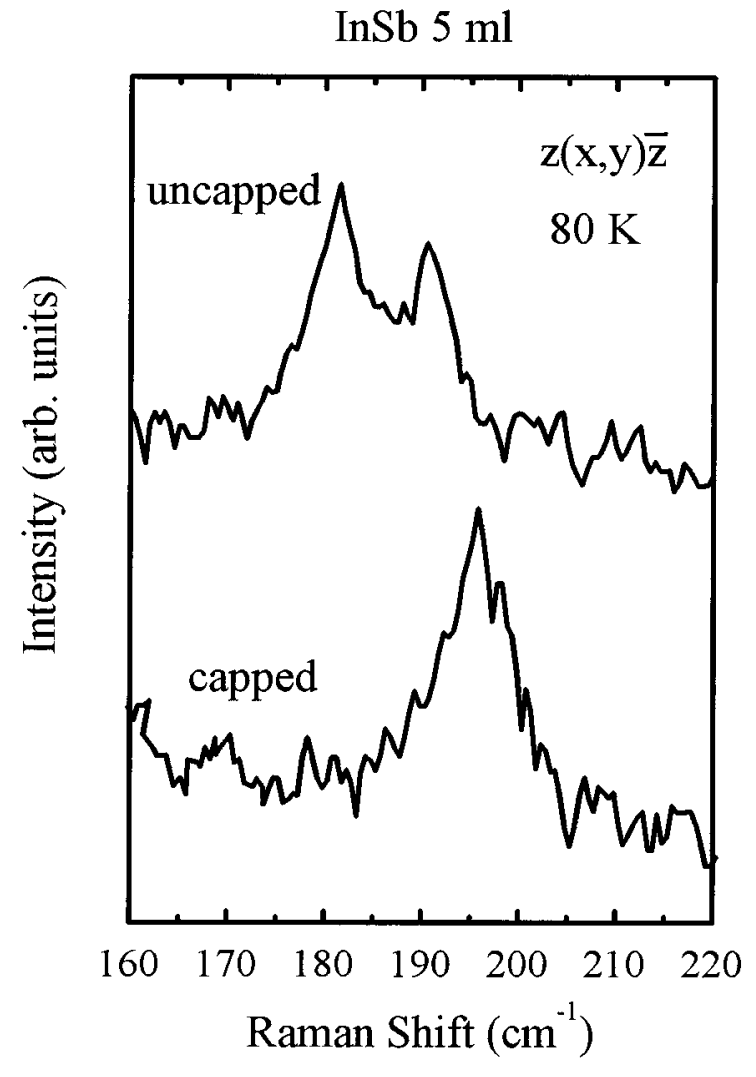

FIG. 4. Raman spectra of samples with and without a cap layer of InP. The TO-like mode disappears for the uncapped samples while the LO-like mode is blue-shifted due to the higher strain in capped QDs. 
systems. ${ }^{9}$ Our dots should be more similar to $\mathrm{CdS}_{1-x} \mathrm{Se}$ nanocrystals embedded in glass. In this system a blue-shift of the phonon frequencies of the nanocrystals has also been observed and attributed to a compressive strain imposed on nanocrystals by the host medium. ${ }^{18}$ If we assume that the buried InSb dots are under a compressive strain, from the shift of the phonon frequencies we obtain a decrease of the InSb lattice parameter in the dots of the sample shown in Fig. 4 of about $0.8 \%$. A very interesting effect is the strong decrease of the TO-like band in the Raman spectra of the capped sample as compared to the Raman spectra of the uncapped sample. The TO scattering is reduced due to the similar value of the refractive index of InSb and InP. Therefore, no backscattering conditions are obtained in the side walls of the buried dots and clear selection rules can be obtained. This confirms our suggestion about the origin of the TO band in the uncapped dots.

InSbP alloys exhibit two mode behaviour, with an InPlike mode located in the frequency range of the InP optical phonons. ${ }^{19}$ No such mode has been detected in our samples, what suggests that the dots are mainly composed of pure InSb. Similar results have been obtained for $\mathrm{GaSb}$ and $\mathrm{InSb}$ dots on GaAs. ${ }^{8}$

\section{CONCLUSION}

In conclusion we have studied the vibrational properties on InSb dots on InP. Two peaks are observed in the Raman spectra of the uncapped dots, corresponding to LO-like and TO-like vibrations of the InSb dots. The results show that the uncapped dots are completely relaxed in agreement with TEM results. In the capped dots only one peak is observed in the Raman spectra, corresponding to the LO-like vibrations of the InSb dots. The shift of the peak to higher frequencies suggest that the buried dots are submitted to a residual strain due to the cap-layer.

\section{ACKNOWLEDGMENTS}

This work has been supported by CICYT under project MAT95-0966 and the HCM program under network CT 330349 .

${ }^{1}$ L. Goldstein, F. Glas, J. Y. Marzin, M. N. Charasse, and G. L. Roux, Appl. Phys. Lett. 47, 1099 (1985).

${ }^{2}$ S. Guha, A. Madhukar and K. C. Rajkumar, Appl. Phys. Lett. 57, 2110 (1990).

${ }^{3}$ J. M. Moison, F. Houzay, F. Barthe, L. Leprince, E. Andre, and O. Vatel, Appl. Phys. Lett. 64, 196 (1994).

${ }^{4}$ D. Leonard, M. Krishnamurthy, C. M. Reaves, S. P. DenBaars, and P. M. Petroff, Appl. Phys. Lett. 63, 3203 (1993).

${ }^{5}$ N. Carlsson, W. Seifert, A. Petersson, P. Castrillo, M.-E. Pistol, and L. Samuelson, Appl. Phys. Lett. 65, 3093 (1994).

${ }^{6}$ B. R. Bennett, R. Magno, and B. V. Shanabrook, Appl. Phys. Lett. 68, 505 (1996).

${ }^{7}$ J. F. Carlin, R. Houdre, A. Rudra, and M. llegems, Appl. Phys. Lett. 59, 3018 (1991)

${ }^{8}$ B. R. Bennett, B. V. Shanabrook, and R. Magno, Appl. Phys. Lett. 68, 958 (1996).

${ }^{9}$ J. Groenen, A. Mlayah, R. Carles, A. Ponchet, A. Lecorre, and S. Salaun, Appl. Phys. Lett. 69, 943 (1996).

${ }^{10}$ M. Grundmann, O. Stier, and D. Bimberg, Phys. Rev. B 52, 11969 (1995).

${ }^{11}$ Y. Gonzalez, L. Gonzalez, and F. Briones, J. Vac Sci. Technol A 13, 73 (1995).

${ }^{12}$ T. Utzmeier, P. A. Postigo, J. Tamayo, R. García, and F. Briones, Appl. Phys. Lett. (in press).

${ }^{13}$ E. Roca, C. Trallero, and M. Cardona, Phys. Rev B 49, 13704 (1994).

${ }^{14}$ F. Cerdeira, C. J. Buchenauer, F. H. Pollak, and M. Cardona, Phys. Rev. B 5, 580 (1972).

${ }^{15}$ Numerical Data and Functional Relationships in Science and Technology, Landolt-Börnstein, New Series, Group III, Vol. 17, Pt a, edited by O. Madelung (Springer, Berlin, 1982).

${ }^{16}$ M. Cardona, in Light Scattering in Solids II, edited by M. Cardona and G. Güntherrodt (Springer, Heidelberg, 1982), p. 45.

${ }^{17}$ M.-E. Pistol, N. Carlsson, C. Persson, W. Seifert, and L. Samuelson, Appl. Phys. Lett. 67, 1438 (1995).

${ }^{18}$ G. Scamarcio, M. Lugara, and D. Manno, Phys. Rev. B 45, 13792 (1992).

${ }^{19}$ M. J. Jou, Y. T. Cherng, and G. B. Stringfellow, J. Appl. Phys. 64, 1472 (1988). 